\title{
Optimal control of discrete-time linear fractional order systems with multiplicative noise
}

\author{
J.J. Trujillo ${ }^{*}$ V.M. Ungureanu ${ }^{\dagger}$
}

\begin{abstract}
A finite horizon linear quadratic(LQ) optimal control problem is studied for a class of discrete-time linear fractional systems (LFSs) affected by multiplicative, independent random perturbations. Based on the dynamic programming technique, two methods are proposed for solving this problem. The first one seems to be new and uses a linear, expanded-state model of the LFS. The LQ optimal control problem reduces to a similar one for stochastic linear systems and the solution is obtained by solving Riccati equations. The second method appeals to the Principle of Optimality and provides an algorithm for the computation of the optimal control and cost by using directly the fractional system. As expected, in both cases the optimal control is a linear function in the state and can be computed by a computer program. Two numerical examples proves the effectiveness of each method.
\end{abstract}

\section{Introduction}

Fractional calculus(FC) began to engage mathematicians' interest in the 17th century as evidenced by a letter of Leibniz to L'Hôspital, dated $30^{\text {th }}$ September 1695 , which talks about the possibility of non-integer order differentiation. Later on, famous mathematicians as Fourier, Euler and Laplace contributed to the foundation of this new branch of mathematics with various concepts and results. Nowadays, the most popular definitions of the non-integer order integral or derivative are the Riemann-Liouville, Caputo and Grunwald-Letnikov definitions. For a historical survey and the current state of the art, the reader is referred to [14, 21], 11, [15, 6] and the references therein.

FC finds use in different fields of science and engineering including the electrochemistry, electromagnetism, biophysics, quantum mechanics, radiation physics, statistics or control theory (see [15], 11], [14]). Such an example comes from the field of autonomous guided vehicles, which lateral control seems to be

\footnotetext{
*Departamento de Análisis Matemático, Universidad de La Laquna, 38271 La Laguna, e-mail jtrujill@ullmat.es

†Department of Mathematics, "Constantin Brancusi" University, Tg. Jiu, Bulevardul Republicii, nr. 1, jud. Gorj, Romaina, e-mail vio@utgjiu.ro.
} 
improved by using fractional adaptation schemes [17. Also, partial differential equations of fractional order were applied to model the wave propagation in viscoelastic media or the dissipation in seismology or in metallurgy [13].

The optimal control theory was intensively developed during the last century for deterministic systems defined by integer-order derivatives, in both continuous- and discrete- time cases [4. Since many real-world phenomena are affected by random factors that exercised a decisive influence on the processes behavior, stochastic optimal control theory had a similar evolution in the recent decades (see [5], [8], [7, [19] and the references therein).

However, only a few papers address optimal control problems for fractional systems (see e.g. [18, [1], 2], [12, [10, 9]) and fewer consider stochastic fractional systems [16, 3].

In this paper we formulate a finite-horizon LQ optimal control problem for stochastic discrete-time LFSs defined by the Grunwald-Letnikov fractional derivative. As far as we know, this subject seems to be new.

We use the classical dynamic programming technique to derive two methods for solving the proposed optimal control problem. Obviously these methods apply to deterministic discrete-time LFSs.

The first one is new and uses an equivalent linear expanded-state model of the stochastic LFS. As the name says, the state of this model is expanded and formed by the actual state and all the past states of the LFS 15. The quadratic cost functional is rewritten accordingly and the original optimal control problem reduces to a LQ optimal control problem for linear stochastic systems. Since the control weight matrix of the new optimization problem is not positive, we modify it with a parameter $\varepsilon>0$ for achieving the positivity condition. This perturbation is chosen such that the optimal value of the new performance index (denoted $I_{x_{0}, N, \varepsilon}(U)$ ) is independent of $\varepsilon$ and coincides with the optimal value of the original cost functional $\left(I_{x_{0}, N}(u)\right)$ (see Proposition 2). The optimal value of the performance index $I_{x_{0}, N, \varepsilon}(U)$ is a quadratic form in the initial expandedstate and can be computed by solving a classical matrix Riccati equation. The optimal feedback law $U$ is linear in state, and involves the solution of the same Riccati equation. The optimal control sequence $u$ of the original optimal control problem is computable from $U$.

The second method is a stochastic version of the new algorithm proposed in [9] for deterministic LFSs. It uses the Optimality Principle for computing recursively (and starting with the terminal time) the optimal control sequence $u_{N-1}, . ., u_{0}$ and the optimal cost.

The main difference between the two methods is that the dynamic programing approach is applied in the first case to a linear stochastic system, while, in the second case, the same technique is applied directly to a stochastic LFS.

To compare the two methods, a numerical example is solved by using two computer algorithms written for this purpose. As expected, the mathematical results are the same, but the run-time of the program that implements the algorithm provided by the first method seems to be shorter.

The paper is organized as follows. In Section 2, we shortly review necessary notions from $\mathrm{FC}$ and we state the optimal control problem $\mathcal{O}$. In Section 3 we 
reformulate the problem $\mathcal{O}$ by using the equivalent linear expanded-state model of the stochastic LFS and a parametrized cost functional $I_{x_{0}, N, \varepsilon}(U)$. As mentioned above, the optimal control and cost can be computed with the solution of an associated Riccati equation. Finally, a numerical example illustrates the effectiveness of this first method.

In Section 4 we present the first two steps of the recursive algorithm (called Algorithm A) which starts with the terminal time and provides the optimal control sequence and cost that solves problem $\mathcal{O}$. The general step of the Algorithm $A$ is described in the Appendix. The numerical example presented at the end in Section 4 is solved by using Algorithm $A$. It proves the applicability of the second method. Some conclusions are drawn in the last section.

\section{Notations and statement of the problem}

As usual, $\mathbb{R}$ is the set of real numbers, $\mathbb{R}^{d}, d \in \mathbb{N}^{*}=\mathbb{N}-\{0\}$ is the real Hilbert space of real $d$-dimensional vectors and $\mathbb{R}^{d \times n}, n \in \mathbb{N}^{*}$ is the linear space of $d \times n$ real matrices. We also denote by $\left(\mathbb{R}^{d}\right)^{n}$ the Hilbert space of all $n$ dimensional vectors from $\mathbb{R}^{d}$. Obviously it is isomorphic with $\mathbb{R}^{d \times n}$. In this paper we do not distinguish between a linear operator on $\mathbb{R}^{d \times n}\left(\right.$ or $\mathbb{R}^{d}$ ) and the associated matrix. Also we shall write $\langle.,$.$\rangle for the inner product and \|$.$\| for norms of$ elements and operators. For any linear operator $T$ acting on finite dimensional real spaces, we denote by $T^{*}$ the adjoint operator of $T$. We say that $T: \mathbb{R}^{n} \rightarrow \mathbb{R}^{n}$ is nonnegative (we write $T \geq 0$ ) if $T=T^{*}$ and $\langle T x, x\rangle \geq 0$, for all $x \in \mathbb{R}^{n} ; T$ is positive (we write $T>0$ ) if $T \geq 0$ and there is $\delta>0$ such that $\langle T x, x\rangle \geq \delta\|x\|^{2}$, for all $x \in \mathbb{R}^{n}$. The identity operator on $\mathbb{R}^{n}$ will be denoted by $I_{\mathbb{R}^{d}}$.

Let $\alpha \in(0,2)$ and $h>0$ be fixed. We recall that for all $j \in \mathbb{N},\left(\begin{array}{c}\alpha \\ j\end{array}\right)$ denotes the generalized binomial coefficient defined by

$$
\left(\begin{array}{c}
\alpha \\
j
\end{array}\right)=\left\{\begin{array}{c}
1, j=0 \\
\frac{\alpha(\alpha-1) \cdot \ldots \cdot(\alpha+1-j)}{j !}, j \in \mathbb{N}^{*}
\end{array} .\right.
$$

Then, for any sequence $\left\{x_{k}\right\}_{k \in \mathbb{N}} \subset \mathbb{R}^{d}, d \in \mathbb{N}$

$$
\Delta^{[\alpha]} x_{k+1}=\frac{1}{h^{\alpha}} \sum_{j=0}^{k+1}(-1)^{j}\left(\begin{array}{c}
\alpha \\
j
\end{array}\right) x_{k+1-j}, h>0
$$

is the discrete fractional-order operator that arises in the Grünwald-Letnikov definition of the fractional order derivatives (see for e.g. [15).

Let $\left\{\xi_{k}\right\}_{k \in \mathbb{N}}$ be a sequence of real-valued, mutually independent random variables on the probability space $(\Omega, \mathcal{G}, P)$ that satisfies the condition $E\left[\xi_{k}\right]=$ $0, E\left[\xi_{k}^{2}\right]=1, k \in \mathbb{N}$. ( Here $E[\xi]$ is the mean (expectation) of $\xi_{k}$.) The $\sigma-$ algebra generated by $\left\{\xi_{i}, 0 \leq i \leq n-1\right\}, n \in \mathbb{N}^{*}$ will be denoted by $\mathcal{G}_{n}$. We 
consider the stochastic discrete-time fractional system with control

$$
\begin{aligned}
\Delta^{[\alpha]} x_{k+1} & =\mathbb{A} x_{k}+\xi_{k} \mathbb{B} x_{k}+\mathbb{D} u_{k}+\xi_{k} \mathbb{F} u_{k}, k \in \mathbb{N} \\
x_{0} & =x \in \mathbb{R}^{d}
\end{aligned}
$$

where $\mathbb{A}, \mathbb{B} \in \mathbb{R}^{d \times d}, \mathbb{D}, \mathbb{F} \in \mathbb{R}^{d \times m}, m \in \mathbb{N}$ and the control $u=\left\{u_{k}\right\}_{k \in \mathbb{N}}$ belongs to a class of admissible controls $\mathcal{U}^{a}$ formed by all sequences $u$ which elements $u_{k}$ are $\mathcal{G}_{k}$-measurable, $\mathbb{R}^{m}$-valued random variables satisfying $E\left[\left\|u_{k}\right\|^{2}\right]<\infty$ for all $k \in \mathbb{N}$.

A finite segment of an admissible control sequence $u$ is of the form $u_{k}, u_{k+1}$, $\ldots, u_{N}$. In the sequel we shall denote by $\mathcal{U}_{k, N-1}^{a}$ the set of segments $u_{k}, u_{k+1}, \ldots$, $u_{N-1}$ of admissible controls $u \in \mathcal{U}^{a}$.

Multiplying (1) by $h^{\alpha}$ and denoting $A_{0}=h^{\alpha} \mathbb{A}+\alpha I_{\mathbb{R}^{d}}, T=h^{\alpha} \mathbb{T}$, for any $T=B, D, F, \mathbb{T}=\mathbb{B}, \mathbb{D}, \mathbb{F}, c_{j}:=(-1)^{j}\left(\begin{array}{c}\alpha \\ j+1\end{array}\right)$ and $A_{j}=c_{j} I_{\mathbb{R}^{d}}$, system (1) can be equivalently rewritten as

$$
\begin{aligned}
x_{k+1} & =\sum_{j=0}^{k} A_{j} x_{k-j}+\xi_{k} B x_{k}+D u_{k}+\xi_{k} F u_{k}, \\
x_{0} & =x \in \mathbb{R}^{d} .
\end{aligned}
$$

Let $x_{0} \in \mathbb{R}^{d}$ and $N \in \mathbb{N}$ be fixed, $C \in \mathbb{R}^{p \times d}, S \in \mathbb{R}^{d \times d}, S \geq 0$ and $K \in$ $\mathbb{R}^{m \times m}, K>0$.

Our optimal control problem $\mathcal{O}$ is to minimize the cost functional

$$
\begin{gathered}
I_{x_{0}, N}(u)= \\
\sum_{n=0}^{N-1} E\left[\left(\left\|C x_{n}\right\|^{2}+<K u_{n}, u_{n}>\right)\right]+E<S x_{N}, x_{N}>
\end{gathered}
$$

subject to (2)-(3), over the class $\mathcal{U}_{0, N-1}^{a}$ of segments of admissible controls.

\section{An equivalent optimal control problem for a non-fractional linear system}

In this section we first present an equivalent linear expanded-state model (see (6) -(77) of the stochastic LFS. Then we show that optimal control problem $\mathcal{O}$ is "equivalent" with a LQ optimal control problem associated with (6)-(7). The word "equivalent" means here that the two optimal control problems have the same optimal costs and an optimal control sequence (OCS) of the one can be obtained from an OCS of the other. Since the solution of the new optimal control problem can be obtained by solving a backward discrete-time Riccati equation, we get a solution of $\mathcal{O}$ (see Theorem 3 ). 


\subsection{A linear expanded-state model}

Let $\mathcal{A}, \mathcal{B}:\left(\mathbb{R}^{d}\right)^{N} \rightarrow\left(\mathbb{R}^{d}\right)^{N}$ be the linear operators defined by the matrices

$$
\mathcal{A}=\left(\begin{array}{cccc}
A_{0} & c_{1} I_{\mathbb{R}^{d}} & \ldots & c_{N-1} I_{\mathbb{R}^{d}} \\
I_{\mathbb{R}^{d}} & 0 & . & 0 \\
\cdot & I_{\mathbb{R}^{d}} & \cdot & \cdot \\
\cdot & \cdot & I_{\mathbb{R}^{d}} & 0
\end{array}\right), \mathcal{B}=\left(\begin{array}{ccccc}
B & 0 & . & . & . \\
0 & 0 & . & . & . \\
. & . & . & \cdot & . \\
. & . & . & . & .
\end{array}\right) .
$$

Also let $\mathcal{D}_{k}, \mathcal{F}_{k}:\left(\mathbb{R}^{m}\right)^{N} \rightarrow\left(\mathbb{R}^{d}\right)^{N}, k=0, . ., N-1$ be given by

$$
\mathcal{D}_{k}\left(v_{0}, v_{1}, \ldots, v_{N-1}\right)=\left(D v_{k}, 0, \ldots, 0\right) \in\left(\mathbb{R}^{d}\right)^{N}
$$

and

$$
\mathcal{F}_{k}\left(v_{0}, v_{1}, \ldots, v_{N-1}\right)=\left(F v_{k}, 0, \ldots, 0\right) \in\left(\mathbb{R}^{d}\right)^{N},
$$

for all $\left(v_{0}, v_{1}, \ldots, v_{N-1}\right) \in\left(\mathbb{R}^{m}\right)^{N}$.

Similarly, for all $k=0, . ., N-1$, we define $\mathcal{K}_{k}:\left(\mathbb{R}^{m}\right)^{N} \rightarrow\left(\mathbb{R}^{m}\right)^{N}, \mathcal{C}:$ $\left(\mathbb{R}^{d}\right)^{N} \rightarrow\left(\mathbb{R}^{p}\right)^{N}$

$$
\begin{aligned}
& \mathcal{K}_{k}\left(v_{0}, v_{1}, \ldots, v_{N-1}\right)=\left(0, \ldots, K v_{k}, 0, \ldots, 0\right) \in \mathbb{R}^{m \times N} \\
& \mathcal{C}\left(v_{0}, v_{1}, \ldots, v_{N}-1\right)=\left(C v_{0}, 0, \ldots, 0\right)
\end{aligned}
$$

and $\mathcal{S}:\left(\mathbb{R}^{d}\right)^{N} \rightarrow\left(\mathbb{R}^{d}\right)^{N}$

$$
\mathcal{S}\left(v_{0}, \ldots, v_{N-1}\right)=\left(S v_{0}, 0, \ldots, 0\right) .
$$

Obviously, $\mathcal{K}_{k}, \mathcal{S} \geq 0$. Let $x_{0}, x_{1}, \ldots, x_{k}, \ldots$ be a solution of (2). For any $k<$ $N, X_{k}^{T}=\left(x_{k}, x_{k-1}, \ldots, x_{0}, 0, . .,{ }_{N}^{0}\right) \in\left(\mathbb{R}^{d}\right)^{N}$ is a solution of the discrete-time system with independent random perturbations

$$
\begin{aligned}
X_{k+1} & =\mathcal{A} X_{k}+\xi_{k} \mathcal{B} X_{k}+\mathcal{D}_{k} U_{k}+\xi_{k} \mathcal{F}_{k} U_{k}, \\
X_{0} & =\left(x_{0}, 0, \ldots,{ }_{N}^{0}\right)
\end{aligned}
$$

where the control $U=\left\{U_{k}\right\}_{k \in \mathbb{N}} \subset\left(\mathbb{R}^{m}\right)^{N}$ belongs to the set $\mathbb{U}^{a}$ of admissible controls sequences $\left\{U_{k}\right\}_{k \in \mathbb{N}}$ having the property that $U_{k}$ are $\left(\mathbb{R}^{m}\right)^{N}$-valued, $\mathcal{G}_{k}$-measurable random variables satisfying $E\left[\left\|U_{k}\right\|^{2}\right]<\infty$ for all $k \in \mathbb{N}$. The system (6)-(7) is a classical linear discrete-time control system with independent random perturbations. We know (see, e.g [8]) that for all $k \in \mathbb{N}^{*}, X_{k}$ is $\mathcal{G}_{k^{-}}$ measurable and the pair $X_{k}, \xi_{n}$ is independent for all $n \geq k>0$.

Computing $X_{N}$ from (6) -(7), we note that $X_{N}=\left(x_{N}, x_{N-1}, \ldots, x_{1}\right)$ and $x_{N}$, the $n$-th solution of (2)-(3), is the first component of $X_{N}$. Then $\left\langle\mathcal{S} X_{N}, X_{N}\right\rangle=$ $\left\langle S x_{n}, x_{n}\right\rangle$. Also, for all $k<N$ we have

$$
\begin{aligned}
\mathcal{C} X_{k} & =\mathcal{C}\left(x_{k}, x_{k-1}, \ldots, x_{0}, 0, . .0\right)=\left(C x_{k}, 0, \ldots, 0\right) \\
\mathcal{K}_{k} U_{k} & =\mathcal{K}_{k}\left(\bar{u}_{0}, \bar{u}_{1}, \ldots, u_{k} . ., \bar{u}_{N-1}\right)=\left(0,0, \ldots, \underset{k+1}{\left.K u_{k}, \ldots, 0\right) .} .\right.
\end{aligned}
$$


Then, the cost functional (4) can be equivalently rewritten as

$$
I_{x_{0}, N}(U)=E\left[\sum_{k=0}^{N-1}\left\langle\mathcal{C}^{*} \mathcal{C} X_{k}, X_{k}\right\rangle+\left\langle\mathcal{S} X_{N}, X_{N}\right\rangle+\left\langle\mathcal{K}_{k} U_{k}, U_{k}\right\rangle\right] .
$$

Substituting $X_{N}$ given by (6)-(17) in (8), we get

$$
\begin{aligned}
I_{x_{0}, N}(U)= & \sum_{n=0}^{N-2} E\left[\left\langle\mathcal{C}^{*} \mathcal{C} X_{k}, X_{k}\right\rangle+\left\langle\mathcal{K}_{k} U_{k}, U_{k}\right\rangle\right] \\
& +E\left[\left\langle\left(\mathcal{C}^{*} \mathcal{C}+\mathcal{A}^{*} \mathcal{S} \mathcal{A}+\mathcal{B}^{*} \mathcal{S B}\right) X_{N-1}, X_{N-1}\right\rangle\right. \\
& +2\left\langle\left(\mathcal{D}_{N-1}^{*} \mathcal{S} \mathcal{A}+\mathcal{F}_{N-1}^{*} \mathcal{S B}\right) X_{N-1}, U_{N-1}\right\rangle+ \\
& \left.\left\langle\left(\mathcal{K}_{N-1}+\mathcal{D}_{N-1}^{*} \mathcal{S D}_{N-1}+\mathcal{F}_{N-1}^{*} \mathcal{S} \mathcal{F}_{N-1}\right) U_{N-1}, U_{N-1}\right\rangle\right]
\end{aligned}
$$

To obtain the last equality we have applied the property of $X_{N-1}$ and $U_{N-1}$ of being independent of $\xi_{N-1}$. Thus for any appropriate deterministic linear operators $V$ and $T$, we have

$$
\begin{gathered}
E\left[\left\langle V X_{N-1}+\xi_{N-1} T U_{N-1}, V X_{N-1}+\xi_{N-1} T U_{N-1}\right\rangle\right]= \\
E\left[\left\langle V X_{N-1}, V X_{N-1}\right\rangle\right]+2 E\left[\xi_{N-1}\right] E\left[\left\langle V X_{N-1}, T U_{N-1}\right\rangle\right]+ \\
E\left[\xi_{N-1}^{2}\right] E\left[\left\langle T U_{N-1}, T U_{N-1}\right\rangle\right] \\
=E\left[\left\langle V X_{N-1}, V X_{N-1}\right\rangle\right]+E\left[\left\langle T U_{N-1}, T U_{N-1}\right\rangle\right]
\end{gathered}
$$

and (9) follows.

Now let $\mathbb{U}_{0, N-1}^{a}$ be the class of all finite segments $U_{0}, \ldots, U_{N-1}$ of sequences $U \in \mathbb{U}^{a}$. It is not difficult to see that the optimal control problem $\mathcal{O}$ is equivalent with the minimizing optimal control problem $\mathcal{O}_{1}$ defined by system (6)-(7), $I_{x_{0}, N}(U)$ and $\mathbb{U}_{0, N-1}^{a}$. Indeed, for any $u \in \mathcal{U}_{0, N-1}^{a}$, the segment $U=\left\{U_{k}=\right.$ $\left.\left(0, . ., u_{k+1}, \ldots 0\right), k=0, . ., N-1\right\}$ belongs to $\mathbb{U}_{0, N-1}^{a}$ and $I_{x_{0}, N}(U)=I_{x_{0}, N}(u)$. Conversely, given $U \in \mathbb{U}_{0, N-1}^{a}$, we define $u=\left\{u_{k}=U_{k k}, k=0, . ., N-1\right\}$. Thus, $u \in \mathcal{U}_{0, N-1}^{a}$ and $I_{x_{0}, N}(U)=I_{x_{0}, N}(u)$. Now it is clear that $\widetilde{U}$ is optimal for $I_{x_{0}, N}(U)$ if and only if $\widetilde{u}=\left\{\widetilde{u}_{k}=\widetilde{U}_{k k}, k=0, . ., N-1\right\}$ is optimal for $I_{x_{0}, N}(u)$ and $I_{x_{0}, N}(\widetilde{U})=I_{x_{0}, N}(\widetilde{u})$.

The problem $\mathcal{O}_{1}$ is a linear quadratic optimal control problem for stochastic systems. However $\mathcal{K}_{k}$ does not satisfy the condition $\mathcal{K}_{k}>0, k=0, . ., N-1$ and we cannot solve $\mathcal{O}_{1}$ by a direct application of the known results from the optimal control theory of stochastic discrete-time systems (see [4, 8]).

Therefore, we replace the optimal cost $I_{x_{0}, N}(U)$ from $\mathcal{O}_{1}$ with the optimal cost

$$
\begin{aligned}
I_{x_{0}, N, \varepsilon}(U)= & \sum_{k=0}^{N-2} E\left[\left\langle\mathcal{C}^{*} \mathcal{C} X_{k}, X_{k}\right\rangle\right]+E\left[\left\langle\left(\mathcal{K}_{k}+\varepsilon \mathcal{I}_{k}\right) U_{k}, U_{k}\right\rangle\right] \\
& +E\left[\left\langle\left(\mathcal{C}^{*} \mathcal{C}+\mathcal{A}^{*} \mathcal{S} \mathcal{A}+\mathcal{B}^{*} \mathcal{S B}\right) X_{N-1}, X_{N-1}\right\rangle\right. \\
& +2\left\langle\left(\mathcal{D}_{N-1}^{*} \mathcal{S} \mathcal{A}+\mathcal{F}_{N-1}^{*} \mathcal{S B}\right) X_{N-1}, U_{N-1}\right\rangle \\
& \left.+\left\langle\mathbb{K}_{N-1}^{\varepsilon} U_{N-1}, U_{N-1}\right\rangle\right]
\end{aligned}
$$


where $\varepsilon>0$ is fixed,

$$
\mathbb{K}_{N-1}^{\varepsilon}=\mathcal{K}_{N-1}+\varepsilon \mathcal{I}_{N-1}+\mathcal{D}_{N-1}^{*} \mathcal{S D}_{N-1}+\mathcal{F}_{N-1}^{*} \mathcal{S} \mathcal{F}_{N-1}
$$

and $\mathcal{I}_{k}\left(v_{0}, v_{1}, \ldots, v_{N-1}\right)=\left(v_{0}, v_{1}, \ldots, \underset{k+1}{0}, \ldots, v_{N-1}\right), k=0, . ., N-1$. We obtain a new optimal control problem $\mathcal{O}_{\varepsilon}$.

The hypothesis $K>0$, implies that $\mathcal{K}_{k}+\varepsilon \mathcal{I}_{k}>0$, for all $k=0, . ., N-1$. Thus we can apply the classical results based on the Principle of Optimality stating that the optimal cost is a quadratic form in the state, with the weighting matrix computable via a recursion that involves the solution of a backward discretetime Riccati equation.

\subsection{Backward discrete-time Riccati equation of control}

We associate with $\mathcal{O}_{\varepsilon}$ the backward discrete-time Riccati equation

$$
\begin{gathered}
R_{n}^{\varepsilon}=\mathcal{A}^{*} R_{n+1}^{\varepsilon} \mathcal{A}+\mathcal{B} R_{n+1}^{\varepsilon} \mathcal{B}+\mathcal{C}^{*} \mathcal{C}-\left(\mathcal{D}_{n}^{*} R_{n+1}^{\varepsilon} \mathcal{A}+\mathcal{F}_{n}^{*} R_{n+1}^{\varepsilon} \mathcal{B}\right)^{*} \\
\left(\mathcal{K}_{n}+\varepsilon \mathcal{I}_{n}+\mathcal{D}_{n}^{*} R_{n+1}^{\varepsilon} \mathcal{D}_{n}+\mathcal{F}_{n}^{*} R_{n+1}^{\varepsilon} \mathcal{F}_{n}\right)^{-1}\left(\mathcal{D}_{n}^{*} R_{n+1}^{\varepsilon} \mathcal{A}+\mathcal{F}_{n}^{*} R_{n+1}^{\varepsilon} \mathcal{B}\right) \\
\quad \text { for } n<N-1 \text { and } \\
R_{N-1}^{\varepsilon}=\mathcal{C}^{*} \mathcal{C}+\mathcal{A}^{*} \mathcal{S} \mathcal{A}+\mathcal{B}^{*} \mathcal{S B}- \\
\left(\mathcal{D}_{N-1}^{*} \mathcal{S} \mathcal{A}+\mathcal{F}_{N-1}^{*} \mathcal{S B}\right)^{*} \cdot\left(\mathbb{K}_{N-1}^{\varepsilon}\right)^{-1}\left(\mathcal{D}_{N-1}^{*} \mathcal{S} \mathcal{A}+\mathcal{F}_{N-1}^{*} \mathcal{S B}\right)
\end{gathered}
$$

Setting

$$
W_{N-1}=-\left(\mathbb{K}_{N-1}^{\varepsilon}\right)^{-1}\left(\mathcal{D}_{N-1}^{*} \mathcal{S} \mathcal{A}+\mathcal{F}_{N-1}^{*} \mathcal{S B}\right),
$$

we observe that

$$
\begin{gathered}
R_{N-1}^{\varepsilon}=\mathcal{C}^{*} \mathcal{C}+\mathcal{A}^{*} \mathcal{S} \mathcal{A}+\mathcal{B}^{*} \mathcal{S B}+\left(\mathcal{D}_{N-1}^{*} \mathcal{S A}+\mathcal{F}_{N-1}^{*} \mathcal{S B}\right)^{*} W_{N-1}+ \\
W_{N-1}^{*}\left(\mathcal{D}_{N-1}^{*} \mathcal{S} \mathcal{A}+\mathcal{F}_{N-1}^{*} \mathcal{S B}\right)+ \\
W_{N-1}^{*}\left(\mathcal{K}_{N-1}+\varepsilon \mathcal{I}_{N-1}+\mathcal{D}_{N-1}^{*} \mathcal{S D}_{N-1}+\mathcal{F}_{N-1}^{*} \mathcal{S} \mathcal{F}_{N-1}\right) W_{N-1} \\
=\mathcal{C}^{*} \mathcal{C}+\left(\mathcal{A}+\mathcal{D}_{N-1} W_{N-1}\right)^{*} \mathcal{S}\left(\mathcal{A}+\mathcal{D}_{N-1} W_{N-1}\right)+ \\
\left(\mathcal{B}+\mathcal{F}_{N-1} W_{N-1}\right)^{*} \mathcal{S}\left(\mathcal{B}+\mathcal{F}_{N-1} W_{N-1}\right)+W_{N-1}^{*}\left(\mathcal{K}_{N-1}+\varepsilon \mathcal{I}_{N-1}\right) W_{N-1}
\end{gathered}
$$

Now it is clear that $R_{N-1}^{\varepsilon} \geq 0$. Denoting

$$
\begin{gathered}
W_{n}=-\left(\mathcal{K}_{n}+\mathcal{D}_{n}^{*} R_{n+1}^{\varepsilon} \mathcal{D}_{n}+\mathcal{F}_{n}^{*} R_{n+1}^{\varepsilon} \mathcal{F}_{n}+\varepsilon I_{n}\right)^{-1} \\
\left(\mathcal{D}_{n}^{*} R_{n+1}^{\varepsilon} \mathcal{A}+\mathcal{F}_{n}^{*} R_{n+1}^{\varepsilon} \mathcal{B}\right), n=0, . ., N-2,
\end{gathered}
$$

and applying formula (4.8) from [20], we obtain

$$
\begin{gathered}
R_{n}^{\varepsilon}=\left(\mathcal{A}+\mathcal{D}_{n} W_{n}\right)^{*} R_{n+1}^{\varepsilon}\left(\mathcal{A}+\mathcal{D}_{n} W_{n}\right)+\left(B+\mathcal{F}_{n} W_{n}\right)^{*} R_{n+1}^{\varepsilon}\left(B+\mathcal{F}_{n} W_{n}\right) \\
+\mathcal{C}^{*} \mathcal{C}+W_{n}^{*}\left(\mathcal{K}_{n}+\varepsilon I_{n}\right) W_{n}, n=0, . ., N-2 .
\end{gathered}
$$

Using the induction, we deduce that Riccati equation (12) has a unique nonnegative solution $R_{n}^{\varepsilon}, n=0, . ., N-1$. 
Lemma 1 The cost functional (10) can be equivalently rewritten as

$$
\begin{gathered}
I_{x_{0}, N, \varepsilon}(U)=E\left[\left\langle R_{0}^{\varepsilon} X_{0}, X_{0}\right\rangle\right]+ \\
\left\langle\mathbb{K}_{N-1}^{\varepsilon}\left(W_{N-1} X_{N-1}-U_{N-1}\right),\left(W_{N-1} X_{N-1}-U_{N-1}\right)\right\rangle+ \\
\sum_{k=0}^{N-2} E\left[\left\|\left(\mathcal{K}_{n}+\mathcal{D}_{n}^{*} R_{n+1}^{\varepsilon} \mathcal{D}_{n}+\mathcal{F}_{n}^{*} R_{n+1}^{\varepsilon} \mathcal{F}_{n}+\varepsilon \mathcal{I}_{n}\right)^{1 / 2}\left(W_{n} X_{n}-U_{n}\right)\right\|^{2}\right],
\end{gathered}
$$

where $R_{n}^{\varepsilon}$ is the unique solution of (12)-(13).

Proof. Let $X_{n+1}$ be defined by ([6). We have

$$
\begin{gathered}
E\left[\left\langle R_{n+1}^{\varepsilon} X_{n+1}, X_{n+1}\right\rangle\right]=E\left[\left\langle\left(\mathcal{A}^{*} R_{n+1}^{\varepsilon} \mathcal{A}+\mathcal{B} R_{n+1}^{\varepsilon} \mathcal{B}\right) X_{n}, X_{n}\right\rangle+\right. \\
2\left\langle\left(\mathcal{D}_{n}^{*} R_{n+1}^{\varepsilon} \mathcal{A}+\mathcal{F}_{n}^{*} R_{n+1}^{\varepsilon} \mathcal{B}\right) X_{n}, U_{n}\right\rangle \\
\left.+\left\langle\left(\mathcal{D}_{n}^{*} R_{n+1}^{\varepsilon} \mathcal{D}_{n}+\mathcal{F}_{n}^{*} R_{n+1}^{\varepsilon} \mathcal{F}_{n}\right) U_{n}, U_{n}\right\rangle\right]
\end{gathered}
$$

and, taking into account (12) and (15), we obtain

$$
\begin{gathered}
E\left[\left\langle R_{n+1}^{\varepsilon} X_{n+1}, X_{n+1}\right\rangle\right]=E\left[\left\langle R_{n}^{\varepsilon} X_{n}, X_{n}\right\rangle-\left\langle\mathcal{C}^{*} \mathcal{C} X_{n}, X_{n}\right\rangle-\right. \\
\left.\left\langle\left(\mathcal{K}_{n}+\varepsilon \mathcal{I}_{n}\right) U_{n}, U_{n}\right\rangle\right]- \\
+E\left[\left\langle\left(\mathcal{K}_{n}+\mathcal{D}_{n}^{*} R_{n+1}^{\varepsilon} \mathcal{D}_{n}+\mathcal{F}_{n}^{*} R_{n+1}^{\varepsilon} \mathcal{F}_{n}+\varepsilon \mathcal{I}_{n}\right) W_{n} X_{n}, W_{n} X_{n}\right\rangle\right. \\
-2 E\left[\left\langle\left(\mathcal{K}_{n}+\mathcal{D}_{n}^{*} R_{n+1}^{\varepsilon} \mathcal{D}_{n}+\mathcal{F}_{n}^{*} R_{n+1}^{\varepsilon} \mathcal{F}_{n}+\varepsilon \mathcal{I}_{n}\right) W_{n} X_{n}, U_{n}\right\rangle\right] \\
+E\left[\left\langle\left(\mathcal{K}_{n}+\mathcal{D}_{n}^{*} R_{n+1}^{\varepsilon} \mathcal{D}_{n}+\mathcal{F}_{n}^{*} R_{n+1}^{\varepsilon} \mathcal{F}_{n}+\varepsilon \mathcal{I}_{n}\right) U_{n}, U_{n}\right\rangle\right] \\
=E\left[\left\langle R_{n}^{\varepsilon} X_{n}, X_{n}\right\rangle-\left\langle\mathcal{C}^{*} \mathcal{C} X_{n}, X_{n}\right\rangle-\left\langle\left(\mathcal{K}_{n}+\varepsilon \mathcal{I}_{n}\right) U_{n}, U_{n}\right\rangle\right] \\
+E\left\langle\left(\mathcal{K}_{n}+\mathcal{D}_{n}^{*} R_{n+1}^{\varepsilon} \mathcal{D}_{n}+\mathcal{F}_{n}^{*} R_{n+1}^{\varepsilon} \mathcal{F}_{n}+\varepsilon \mathcal{I}_{n}\right)\left(W_{n} X_{n}-U_{n}\right),\left(W_{n} X_{n}-U_{n}\right)\right\rangle .
\end{gathered}
$$

for all $n=0, . ., N-2$. Summing for $n=0$ to $N-2$ the last equality, we obtain

$$
\begin{gathered}
E\left[\left\langle R_{N-1}^{\varepsilon} X_{N-1}, X_{N-1}\right\rangle\right]=E\left[\left\langle R_{0}^{\varepsilon} X_{0}, X_{0}\right\rangle\right]- \\
\sum_{k=0}^{N-2} E\left[\left\langle\mathcal{C}^{*} \mathcal{C} X_{k}, X_{k}\right\rangle\right]+E\left[\left\langle\left(\mathcal{K}_{k}+\varepsilon \mathcal{I}_{k}\right) U_{k}, U_{k}\right\rangle\right] \\
+\sum_{k=0}^{N-2} E\left[\left\|\left(\mathcal{K}_{n}+\mathcal{D}_{n}^{*} R_{n+1}^{\varepsilon} \mathcal{D}_{n}+\mathcal{F}_{n}^{*} R_{n+1}^{\varepsilon} \mathcal{F}_{n}+\varepsilon \mathcal{I}_{n}\right)^{1 / 2}\left(W_{n} X_{n}-U_{n}\right)\right\|^{2}\right] .
\end{gathered}
$$

On the other hand, from (13), (11) and (14), we know that

$$
\begin{gathered}
E\left[\left\langle R_{N-1}^{\varepsilon} X_{N-1}, X_{N-1}\right\rangle\right]=E\left[\left\langle\left(\mathcal{C}^{*} \mathcal{C}+\mathcal{A}^{*} \mathcal{S} \mathcal{A}+\mathcal{B}^{*} \mathcal{S B}\right) X_{N-1}, X_{N-1}\right\rangle\right. \\
\left.-\left\langle\mathbb{K}_{N-1}^{\varepsilon} W_{N-1} X_{N-1}, W_{N-1} X_{N-1}\right\rangle\right] \\
=E\left[\left\langle\left(\mathcal{C}^{*} \mathcal{C}+\mathcal{A}^{*} \mathcal{S} \mathcal{A}+\mathcal{B}^{*} \mathcal{S B}\right) X_{N-1}, X_{N-1}\right\rangle\right. \\
-\left\langle\mathbb{K}_{N-1}^{\varepsilon}\left(W_{N-1} X_{N-1}-U_{N-1}\right),\left(W_{N-1} X_{N-1}-U_{N-1}\right)\right\rangle \\
\left.+2\left\langle\left(\mathcal{D}_{N-1}^{*} \mathcal{S} \mathcal{A}+\mathcal{F}_{N-1}^{*} \mathcal{S B}\right) X_{N-1}, U_{N-1}\right\rangle+\left\langle\mathbb{K}_{N-1}^{\varepsilon} U_{N-1}, U_{N-1}\right\rangle\right] .
\end{gathered}
$$

Replacing the above formula in (18), we obtain (16) and the conclusion follows. 


\subsection{Main results}

In this section we shall prove that problem $\mathcal{O}$ has a solution derived from the solution of problem $\mathcal{O}_{\varepsilon}$.

Proposition 2 For all $\varepsilon>0$

$$
\min _{U \in \mathbb{U}_{0, N-1}^{a}} I_{x_{0}, N, \varepsilon}(U)=\min _{u \in \mathcal{U}_{0, N-1}^{a}} I_{x_{0}, N}(u) .
$$

Proof. Let $u=\left\{u_{0}, . ., u_{N-1}\right\} \in \mathcal{U}_{0, N-1}^{a}$. If $U=\left\{U_{0}, . ., U_{N-1}\right\}$,

$$
\begin{gathered}
U_{k}=\left(0, . ., u_{k}, \ldots 0\right) \text {, then } U \in \mathbb{U}_{0, N-1}^{a} \text { and } I_{x_{0}, N, \varepsilon}(U)=I_{x_{0}, N}(u) \text {. Thus } \\
\min _{u \in \mathcal{U}_{0, N-1}^{a}} I_{x_{0}, N}(u) \geq \min _{U \in \mathbb{U}} I_{x_{0}, N, \varepsilon}(U) .
\end{gathered}
$$

On the other hand if $U \in \mathbb{U}_{0, N-1}^{a}$ and $u=\left\{u_{0}, . ., u_{N-1}\right\}$ is defined by $u_{k}=$ $U_{k k}, k=0,1, . ., N-1$, then $u \in \mathcal{U}_{0, N-1}^{a}$ and $I_{x_{0}, N}(u)=I_{x_{0}, N}(U) \leq I_{x_{0}, N, \varepsilon}(U)$. Replacing $U$ in the above inequality by $\widetilde{U}^{\varepsilon}$ the optimal control which minimizes $I_{x_{0}, N, \varepsilon}(U)$ (we know that it exists), we see that $I_{x_{0}, N}(\widetilde{u}) \leq I_{x_{0}, N, \varepsilon}\left(\widetilde{U}^{\varepsilon}\right)=$ $\min _{U \in \mathbb{U}_{0, N-1}^{a}} I_{x_{0}, N, \varepsilon}(U)$, where $\widetilde{u}=\left\{\widetilde{u}_{0}, . ., \widetilde{u}_{N-1}\right\}$ and $\widetilde{u}_{k}=\widetilde{U}_{k k}$. Therefore $\min _{u \in \mathcal{U}_{0, N-1}^{a}} I_{x_{0}, N}(u) \leq \min _{U \in \mathbb{U}_{0, N-1}^{a}} I_{x_{0}, N, \varepsilon}(U)$. In view of (19) we get the conclusion.

The next theorem is a direct consequence of Lemma 1 and of the above proposition.

Theorem 3 Let $\left\{R_{n}^{\varepsilon}\right\}_{n=0, . ., N-1}$ be the unique solution of the Riccati equation (12)-(13) and let $W_{n}, n=0, . ., N-1$ be defined by (15), (14). The control sequence $\widetilde{U}=\left\{\widetilde{U}_{0}=W_{0} X_{0}, \ldots, \widetilde{U}_{n}=W_{n} X_{n}, \ldots, \widetilde{U}_{N-1}=W_{N-1} X_{N-1}\right\}$ minimizes the cost functional $I_{x_{0}, N, \varepsilon}(U)$ and $\min _{U \in \mathbb{U}_{0, N-1}^{a}} I_{x_{0}, N, \varepsilon}(U)=E\left[\left\langle R_{0}^{\varepsilon} X_{0}, X_{0}\right\rangle\right]$.

Moreover, $\left\{R_{n}^{\varepsilon}\right\}_{n=0, . ., N-1}$ does not depend on $\varepsilon$ and the control

$\widetilde{u}=\left\{\widetilde{u}_{0}, \ldots, \widetilde{u}_{N-1}\right\}$, defined by $\widetilde{u}_{k}=\widetilde{U}_{k k}, k=0,1, . ., N-1$ is also optimal for $I_{x_{0}, N}(u)$. The optimal cost is

$$
\min _{u \in \mathcal{U}_{0, N-1}^{a}} I_{x_{0}, N}(u)=E\left[\left\langle R_{0}^{\varepsilon} X_{0}, X_{0}\right\rangle\right] .
$$

Proof. The proof is a simple exercise for the reader.

The following numerical example illustrates the applicability of the theory.

Example 4 Let $\alpha=\frac{1}{2}, h=1, d=2, m=1, x_{0}=\left(\begin{array}{l}0.2 \\ 0.3\end{array}\right)$ and $\mathbb{A}=\left(\begin{array}{ll}1 & 0 \\ 1 & 0\end{array}\right)$, $\mathbb{B}=\left(\begin{array}{ll}1 & 2 \\ 0 & 1\end{array}\right), \mathbb{D}=\left(\begin{array}{c}1 \\ -1\end{array}\right), \mathbb{F}=\left(\begin{array}{l}2 \\ 1\end{array}\right), C=\left(\begin{array}{ll}2 & -1\end{array}\right), K=1, S=$ $\left(\begin{array}{ll}2 & 0 \\ 0 & 2\end{array}\right)$. Then $A_{0}=\left(\begin{array}{cc}3 / 2 & 0 \\ 1 & 1 / 2\end{array}\right)$ and $T=\mathbb{T}$ for $\mathbb{T}=\mathbb{B}, \mathbb{D}, \mathbb{F}$. We consider the optimal control problem $\mathcal{O}$ for $N=4$. Using a computer program, 
we compute in four simple steps the solution $R_{0}^{\varepsilon}$ of the Riccati equation (12)(13). The first five lines and columns of the matrix that defines the operator $R_{0}^{\varepsilon}$ are the following

$$
\left(\begin{array}{cccccc}
266.5781 & 33.3776 & 14.1358 & 16.6723 & 7.5490 & . \\
33.3776 & 149.0853 & -9.7899 & 8.6506 & -4.8235 & . \\
14.1358 & -9.7899 & 1.7822 & 0.4176 & 0.9168 & . \\
16.6723 & 8.6506 & 0.4176 & 1.3910 & 0.2413 & . \\
7.5490 & -4.8235 & 0.9168 & 0.2413 & 0.4730 & . \\
. & . & . & . & . & .
\end{array}\right)
$$

Using the intermediate values $R_{n}^{\varepsilon}$, (15) and Theorem 3, we obtain the following optimal control sequence

$$
\begin{aligned}
\widetilde{u}_{3}= & -0.3333 x_{31}-0.6000 x_{32}-0.0167 x_{21}+0.0167 x_{22}- \\
& 0.0083 x_{11}+0.0083 x_{12}-0.0052 x_{01}+0.0052 x_{00} \\
\widetilde{u}_{2}= & -0.4788 x_{21}-0.6894 x_{22}-0.0234 x_{11}+0.0109 x_{12}- \\
& 0.0121 x_{01}+0.0054 x_{02} \\
\widetilde{u}_{1}= & -0.4563 x_{11}-0.7023 x_{12}-0.0253 x_{01}+0.0107 x_{02} \\
\widetilde{u}_{0}= & -0.4582 x_{01}-0.7484 x_{02},
\end{aligned}
$$

where $x_{n}=\left(\begin{array}{l}x_{n 1} \\ x_{n 2}\end{array}\right), n=1,2,3$ is the state vector of the fractional system. The optimal cost is

$$
\min _{u \in \mathcal{U}_{0, N-1}^{a}} I_{x_{0}, N}(u)=x_{0}^{T}\left(\begin{array}{cc}
266.5781 & 33.3776 \\
33.3776 & 149.0853
\end{array}\right) x_{0}=28.086 .
$$

\section{A dynamic programming approach for the fractional system}

In this section we apply the Principle of Optimality to derive a direct algorithm for solving the optimal control problem $\mathcal{O}$. As in 9 , the optimal control is a state feedback law, computable via a recursion commencing at the terminal time and evolving backwards. The obtained result is a stochastic counterpart of the one provided in [9] for deterministic fractional systems.

\section{Algorithm A}

Consider the optimal control problem $\mathcal{O}$. An optimal control process $P_{0}$ is defined by the control policy $u=\left\{u_{k}\right\}_{k \in\{0, . ., N-1\}}$ and the corresponding trajectory $x=\left\{x_{k}\right\}_{k \in\{0, . ., N\}}$. Let

$$
\begin{aligned}
P_{m}: & x_{m}, x_{m+1} . ., x_{N-1}, x_{N} \\
& u_{m}, . ., u_{N-2}, u_{N-1} .
\end{aligned}
$$


be a final segment of $P_{0}$ starting at a time $t=m$, when system (2) is in the state $x_{m}$ obtained from the initial state $x_{0}$ with the optimal control sequence $u_{0}, . ., u_{m-1}$. The performance functional on this final segment is

$$
\begin{gathered}
I_{m, x_{0}, . ., x_{m}}(u)= \\
\sum_{n=m}^{N-1} E\left[\left(\left\|C x_{n}\right\|^{2}+<K u_{n}, u_{n}>\right)\right]+E<S x_{N}, x_{N}>.
\end{gathered}
$$

The Principle of Optimality says that any final segment $P_{m}$ of $P_{0}$ must be optimal for $I_{m, x_{0}, . ., x_{m}}$.

Thus for $m=N-1$, the process

$$
\begin{aligned}
P_{N-1}: & x_{N-1}, x_{N} \\
& u_{N-1}
\end{aligned}
$$

should be optimal for the cost

$$
\begin{aligned}
I_{N-1, x_{0}, \ldots, x_{N-1}}(u)= & E\left[\left(\left\|C x_{N-1}\right\|^{2}+\left\langle K u_{N-1}, u_{N-1}\right\rangle\right)\right]+ \\
& E\left[\left\langle S x_{N}, x_{N}\right\rangle\right] .
\end{aligned}
$$

This condition and the following computations leads to a formula for the optimal control $u_{N-1}$. Writing (2) for $k=N-1$, we obtain $x_{N}$. Substituting $x_{N}$ in (25), we get

$$
\begin{gathered}
I_{N-1, x_{0}, . ., x_{N-1}}(u)= \\
=E<S\left(\sum_{j=0}^{N-1} A_{j} x_{N-1-j}+\xi_{N-1} B x_{N-1}+D u_{N-1}+\xi_{N-1} F u_{N-1}\right), \\
\sum_{j=0}^{N-1} A_{j} x_{N-1-j}+\xi_{N-1} B x_{N-1}+D u_{N-1}+\xi_{N-1} F u_{N-1}>+ \\
+E\left[\left(\left\|C x_{N-1}\right\|^{2}+<K u_{N-1}, u_{N-1}>\right)\right]
\end{gathered}
$$

Since $x_{n}, u_{n}$ are $\mathcal{G}_{n}$-measurable and $\xi_{p}$-independent for all $p \geq n, n, p \in \mathbb{N}$, we have

$$
\begin{aligned}
E\left[\left\langle T x_{i}, \xi_{n} S v_{n},\right\rangle\right] & =E\left[\xi_{n}\right] E\left[\left\langle T x_{i}, S v_{n},\right\rangle\right]=0 \\
E\left[\left\langle\xi_{n} T v_{n}, \xi_{n} S v_{n},\right\rangle\right] & =E\left[\xi_{n}^{2}\right] E\left[\left\langle T v_{n}, S v_{n},\right\rangle\right]=E\left[\left\langle T v_{n}, S v_{n},\right\rangle\right]
\end{aligned}
$$


for all $i \leq n, v=u, x$ and $S, T$ matrices of appropriate dimensions. Therefore,

$$
\begin{gathered}
I_{N-1, x_{0}, . ., x_{N-1}}(u)=E\left\langle S \sum_{j=0}^{N-1} A_{j} x_{N-1-j}, \sum_{j=0}^{N-1} A_{j} x_{N-1-j}\right\rangle+ \\
E\left\langle S B x_{N-1}, B x_{N-1}\right\rangle+2 E\left\langle D^{*} S \sum_{j=0}^{N-1} A_{j} x_{N-1-j}, u_{N-1}\right\rangle+ \\
E\left\langle F^{*} S F u_{N-1}, u_{N-1}\right\rangle+2 E\left\langle F^{*} S B x_{N-1}, u_{N-1}\right\rangle \\
+E\left\langle D^{*} S D u_{N-1}, u_{N-1}\right\rangle+E\left[\left(\left\|C x_{N-1}\right\|^{2}+\left\langle K u_{N-1}, u_{N-1}\right\rangle\right)\right] \\
=E\left[\left\|\sqrt{S} \sum_{j=0}^{N-1} A_{j} x_{N-1-j}\right\|^{2}\right]+E\left[\left\|\sqrt{S} B x_{N-1}\right\|^{2}\right]+E\left[\left\|C x_{N-1}\right\|^{2}\right] \\
+2 E\left\langle D^{*} S \sum_{j=1}^{N-1} A_{j} x_{N-1-j}+\left(F^{*} S B+D^{*} S A_{0}\right) x_{N-1}, u_{N-1}\right\rangle \\
+E\left\langle\left(F^{*} S F+D^{*} S D+K\right) u_{N-1}, u_{N-1}\right\rangle .
\end{gathered}
$$

Setting

$$
\begin{gathered}
v_{N-1}\left(x_{0}, . ., x_{N-1}\right)=D^{*} S \sum_{j=1}^{N-1} A_{j} x_{N-1-j}+\left(F^{*} S B+D^{*} S A_{0}\right) x_{N-1}, \\
w_{N-1}\left(x_{0}, . ., x_{N-1}\right)=E\left[\left\|\sqrt{S} \sum_{j=0}^{N-1} A_{j} x_{N-1-j}\right\|^{2}\right] \\
+E\left[\left\|\sqrt{S} B x_{N-1}\right\|^{2}\right]+E\left[\left\|C x_{N-1}\right\|^{2}\right] \\
J_{N-1}=F^{*} S F+D^{*} S D+K>0
\end{gathered}
$$

and using a squares completion technique, we see that

$$
\begin{gathered}
I_{N-1, x_{0}, . ., x_{N-1}}(u)=w_{N-1}\left(x_{0}, . ., x_{N-1}\right)+ \\
2 E\left\langle v_{N-1}\left(x_{0}, . ., x_{N-1}\right), u_{N-1}\right\rangle+E\left\langle J_{N-1} u_{N-1}, u_{N-1}\right\rangle .
\end{gathered}
$$

The cost functional $I_{N-1, x_{0}, . ., x_{N-1}}(u)$ can be equivalently rewritten as

$$
\begin{aligned}
I_{N-1, x_{0}, \ldots, x_{N-1}}(u)= & E\left\langle J_{N-1}\left(u_{N-1}+J_{N-1}^{-1} v_{N-1}\right),\left(u_{N-1}+J_{N-1}^{-1} v_{N-1}\right)\right\rangle \\
& +w_{N-1}-E\left\langle J_{N-1}^{-1} v_{N-1}, v_{N-1}\right\rangle .
\end{aligned}
$$

As a function of $u_{N-1}, I_{N-1, x_{0}, \ldots, x_{N-1}}(u)$ is optimal for

$$
u_{N-1}^{*}=-J_{N-1}^{-1} v_{N-1}
$$


and its optimal value is

$$
\min _{u_{N-1} \in \mathcal{U}_{N-1, N-1}^{a}} I_{N-1, x_{0}, . ., x_{N-1}}(u)=w_{N-1}-E\left\langle J_{N-1}^{-1} v_{N-1}, v_{N-1}\right\rangle .
$$

In view of (27),

$$
u_{N-1}^{*}\left(x_{0}, . ., x_{N-1}\right)=\sum_{j=0}^{N-1} W_{j, N-1} x_{N-1-j},
$$

where

$$
\begin{aligned}
& W_{j, N-1}=-\left(F^{*} S F+D^{*} S D+K\right)^{-1} D^{*} S A_{j}, j \in\{1, . ., N-1\} \\
& W_{0, N-1}=-\left(F^{*} S F+D^{*} S D+K\right)^{-1}\left(F^{*} S B+D^{*} S A_{0}\right) .
\end{aligned}
$$

From the above proof we deduce that $u_{N-1}^{*}\left(x_{0}, . ., x_{N-1}\right)=\sum_{j=0}^{N-1} W_{j, N-1} x_{N-1-j}$ is optimal for $I_{N-1}(u)$ for any trajectory $\left(x_{0}, . ., x_{N-1}\right)$. Substituting (28) to (26) we obtain

$$
\begin{gathered}
\min _{u_{N-1} \in \mathcal{U}_{N-1, N-1}^{a}} I_{N-1, x_{0}, . ., x_{N-1}}(u)=\mathcal{O}\left(x_{0}, . ., x_{N-1}\right)= \\
E<S\left(\sum_{j=0}^{N-1} A_{j} x_{N-1-j}+\xi_{N-1} B x_{N-1}+\left(D+\xi_{N-1} F\right) \sum_{j=0}^{N-1} W_{j, N-1} x_{N-1-j}\right), \\
\left.\sum_{j=0}^{N-1} A_{j} x_{N-1-j}+\xi_{N-1} B x_{N-1}+\left(D+\xi_{N-1} F\right) \sum_{j=0}^{N-1} W_{j, N-1} x_{N-1-j}\right)>+ \\
+E\left[\left\|C x_{N-1}\right\|^{2}\right]+E\left[<K \sum_{j=0}^{N-1} W_{j, N-1} x_{N-1-j}, \sum_{j=0}^{N-1} W_{j, N-1} x_{N-1-j}>\right] \\
=E<S\left(\sum_{j=0}^{N-1} V_{N-1, j}^{S, 1} x_{N-1-j}+\xi_{N-1} \sum_{j=0}^{N-1} V_{N-1, j}^{S, 2} x_{N-1-j}\right), \\
+E\left[\left\|C x_{N-1}\right\|^{2}\right]+E\left[<K \sum_{j=0}^{N-1} V_{N-1, j}^{S, 1} x_{N-1-j}+\xi_{N-1} \sum_{j=0}^{N-1} V_{N-1, j}^{S, 2} x_{N-1-j}>+\right. \\
\left.\sum_{j=0} x_{N-1-j}, \sum_{j=0}^{N-1} W_{j, N-1} x_{N-1-j}>\right]
\end{gathered}
$$

Denoting

$$
\begin{aligned}
& V_{N-1,0}^{S, 2}=B+F W_{0, N-1}, V_{N-1, j}^{S, 2}=F W_{j, N-1}, j \neq 0 \\
& V_{N-1, j}^{S, 1}=A_{j}+D W_{j, N-1}, V_{N-1, j}^{K, 1}=W_{j, N-1}, j \in\{0, . ., N-1\}
\end{aligned}
$$


we obtain the optimal value of the cost:

$$
\begin{gathered}
\mathcal{O}\left(x_{0}, . ., x_{N-1}\right)=E\left[\left\|\sqrt{S} \sum_{j=0}^{N-1} V_{N-1, j}^{S, 1} x_{N-1-j}\right\|^{2}\right]+ \\
E\left[\left\|\sqrt{S} \sum_{j=0}^{N-1} V_{N-1, j}^{S, 2} x_{N-1-j}\right\|^{2}\right]+ \\
E\left[\left\|C x_{N-1}\right\|^{2}\right]+E\left[\left\|\sqrt{K} \sum_{j=0}^{N-1} V_{N-1, j}^{K, 1} x_{N-1-j}\right\|^{2}\right]
\end{gathered}
$$

Now we assume that

$$
\begin{aligned}
P_{N-2}: & x_{N-2}, x_{N-1}, x_{N} \\
& u_{N-2}, u_{N-1}
\end{aligned}
$$

is a final segment of the process $P_{0}$.

Then $P_{N-2}$ should be optimal for $I_{N-2, x_{0}, . ., x_{N-2}}(u)$. Since

$$
\begin{gathered}
\min _{u_{N-2}, u_{N-1} \in \mathcal{U}_{N-2, N-1}^{a}} I_{N-2, x_{0}, . ., x_{N-2}}(u)= \\
\min _{u_{N-2}, u_{N-1} \in \mathcal{U}_{N-2, N-1}^{a}}\left\{I_{N-1, x_{0}, . ., x_{N-1}}(u)+E\left[\left\|C x_{N-2}\right\|^{2}\right]+\right. \\
\left.E\left[\left\langle K u_{N-2}, u_{N-2}\right\rangle\right]\right\}= \\
\min _{u_{N-2} \in \mathcal{U}_{N-2, N-2}^{a}}\left\{\min _{u_{N-1} \in \mathcal{U}_{N-1, N-1}^{a}} I_{N-1, x_{0}, . ., x_{N-1}}(u)+E\left[\left\|C x_{N-2}\right\|^{2}\right]+\right. \\
\left.+E\left[\left\langle K u_{N-2}, u_{N-2}\right\rangle\right]\right\}= \\
\left.\min _{u_{N-2} \in \mathcal{U}_{N-2, N-2}^{a}}\left\{\mathcal{O}\left(x_{0}, . ., x_{N-1}\right)+E\left[\left\|C x_{N-2}\right\|^{2}+<K u_{N-2}, u_{N-2}\right\rangle\right]\right\},
\end{gathered}
$$

it follows that $u_{N-1}$ is given by (30) and $u_{N-2}$ should be computed. Substituting $x_{N-1}$ given by (2) in $\mathcal{O}\left(x_{0}, . . x_{N-2}, x_{N-1}\right)$, we see that $\mathcal{O}\left(x_{0}, . . x_{N-2}, x_{N-1}\right)=$ $\phi\left(x_{0}, . . x_{N-2}, u_{N-2}\right)$ and $u_{N-2}$ solves the optimal control problem

$$
\begin{gathered}
\min _{u_{N-2}, u_{N-1} \in \mathcal{U}_{N-2, N-1}^{a}} I_{N-2, x_{0}, . ., x_{N-2}}(u)= \\
=\min _{u_{N-2} \in \mathcal{U}_{N-2, N-2}^{a}}\left\{\phi\left(x_{0}, . . x_{N-2}, u_{N-2}\right)+E\left[\left(\left\|C x_{N-2}\right\|^{2}+<K u_{N-2}, u_{N-2}>\right)\right]\right\} .
\end{gathered}
$$

Using again the squares completion technique, we can prove that the optimal control $u_{N-2}$ is a linear function of $x_{0}, . . x_{N-2}$ and $\min _{u_{N-2}, u_{N-1} \in \mathcal{U}_{N-2, N-1}^{a}} I_{N-2}(u)$ is a function of the trajectory $x_{0}, . ., x_{N-2}$. Repeating the above arguments, we find $u_{N-3}, u_{N-4}$ and so on. The general step of the above algorithm is 
described in detail in the Appendix. At the step $q$ we find the optimal control $u_{N-q}$ as a linear function of $x_{0}, . ., x_{N-q}$ of the form $\sum_{j=0}^{N-q} W_{j, N-q} x_{N-q-j}$ where the coefficients $W_{j, N-q}$ are given by a set of recurrent formulas (see (36), (38), (39),(40) in the Appendix). At a first sight this algorithm is more complicated than the one described in Section 3.

Example 5 Consider the optimal control problem $\mathcal{O}$ under the hypotheses of Example 4. Implementing in MATLAB the Algorithm A, we obtain the following results. Since $W_{0,3}=\left(\begin{array}{ll}-0.3333 & -0.600\end{array}\right), W_{1,3}=\left(\begin{array}{ll}-0.0167 & 0.0167\end{array}\right)$, $W_{2,3}=\left(\begin{array}{ll}-0.0083 & 0.0083\end{array}\right)$ and $W_{3,3}=\left(\begin{array}{ll}-0.0052 & 0.0052\end{array}\right)$ we deduce by (30) that the optimal control $u_{3}$ have the same formula as the one obtained in Example 4. Further, we compute $V_{3, j}^{S, 1}, V_{3, j}^{S, 2}, V_{3, j}^{K, 1}, j=0,1,2,3$. Writing (35) for $q=2$ we get $J_{2}=67.3667$. Also the coefficients of $x_{0}, x_{1}$ and $x_{2}$ from (36) are $\left(\begin{array}{ll}0.8151 & -0.3630\end{array}\right),\left(\begin{array}{ll}1.5750 & -0.7333\end{array}\right)$ and ( $\left.\begin{array}{ll}32.2583 & 46.4417\end{array}\right)$, respectively. Since $W_{j, N-q}$ is obtained by multiplying the coefficient of $x_{N-q-j}$ from $v_{N-q}$ with $-J_{N-q}^{-1}$, we get $W_{0,2}=-J_{2}^{-1}\left(\begin{array}{ll}32.2583 & 46.4417\end{array}\right)=\left(\begin{array}{ll}-0.47885 & -0.68939\end{array}\right)$, $W_{1,2}=\left(\begin{array}{ll}-0.0233 & 0.0108\end{array}\right)$ and $W_{2,2}=\left(\begin{array}{ll}-0.0120 & 0.0053\end{array}\right)$. Thus

$$
\begin{aligned}
u_{2}= & \left(\begin{array}{ll}
-0.47885 & -0.68939
\end{array}\right) x_{2}+\left(\begin{array}{ll}
-0.0233 & 0.0108
\end{array}\right) x_{1}+ \\
& \left(\begin{array}{ll}
-0.0120 & 0.0053
\end{array}\right) x_{0}
\end{aligned}
$$

and the formula of $\widetilde{u}_{2}$ obtained in Example 4 is recovered. At the next step are computed the coefficients $V_{3, j}^{S, l}, l=1, . ., 4, V_{3, j}^{S, l}, l=1,2,3, V_{3, j}^{K, 1}, l=1,2, j=$ $0,1,2$. With (35) written for $q=1$ we obtain $J_{1}=196.1711$. The coefficients of $x_{0}$ and $x_{1}$ from (36) are $\left(\begin{array}{lll}-0,0253 & 0.0107\end{array}\right),\left(\begin{array}{ll}4.8670 & -2.2318\end{array}\right)$ and

$$
u_{1}=\left(\begin{array}{ll}
-0.45634 & -0.70234
\end{array}\right) x_{1}+\left(\begin{array}{ll}
-0,0253 & 0.0107
\end{array}\right) x_{0} .
$$

Continuing the procedure, we obtain $u_{0}=\left(\begin{array}{ll}-0.4589 & -0.7481\end{array}\right)$ and $\mathcal{O}\left(x_{0}\right)=$ $x_{0}^{T}\left(\begin{array}{cc}266.8471 & 32.9452 \\ 32.9452 & 149.4033\end{array}\right) x_{0}=28.074$.

\section{Conclusions}

This paper provides two methods of solving the LQ optimal control problem $\mathcal{O}$. Both of them are based on the dynamic programming approach. The first one seems to be new and easier. It consists in a reformulation of the problem for an associated linear non-fractional system (6)-(7), defined on spaces of higher dimensions. The second one uses the Principle of Optimality to derive a dynamic programming algorithm for the optimal control of the LFS. This algorithm is a stochastic counterpart of the one obtained in [9 for deterministic LFSs; it keeps the dimensions of the state space of system (2)-(3), but it is more laborious. The computer program implementing it is not such simple and fast as the one that implements the first method. A future analysis of these algorithms from the computer science point of view will highlight the real advantages and disadvantages of each method. 


\section{Appendix}

\section{The general step of the algorithm $A$}

Our problem is to find the final segment

$$
\begin{aligned}
P_{N-q}: & x_{N-q}, x_{N-q+1} ., x_{N-1}, x_{N} \\
& u_{N-q}, . ., u_{N-2}, u_{N-1} .
\end{aligned}
$$

of $P_{0}$ which minimizes $I_{N-q, x_{0}, . ., x_{N-q}}(u)$. Assume that the optimal controls $u_{N-1}, \ldots, u_{N-q+1}, q \geq 2$ were determined and the optimal cost

$$
I_{N-q+1,, x_{0}, \ldots, x_{N-q+1}}(u)
$$

has the form

$$
\begin{gathered}
\mathcal{O}\left(x_{0}, . . x_{N-q}, x_{N-q+1}\right):=\min _{u_{N-q+1}, \ldots, u_{N-1} \in \mathcal{U}_{N-q+1, N-1}^{a}} I_{N-q+1, x_{0}, . ., x_{N-q+1}}(u)= \\
\sum_{l=1}^{2^{q-1}} E\left\|\sqrt{S} \sum_{j=0}^{N-q+1} V_{N-q+1, j}^{S, l} x_{N-q+1-j}\right\|^{2}+ \\
\sum_{l=1}^{2^{q-1}-1} E\left[\left\|\sqrt{K} \sum_{j=0}^{N-q+1} V_{N-q+1, j}^{K, l} x_{N-q+1-j}\right\|^{2} \|^{2}\right. \\
\sum_{l=1}^{2^{q-1}-2} E\left[\left\|\sum_{j=0}^{N-q+1} V_{N-q+1, j}^{C, l} x_{N-q+1-j}\right\|^{2} E\left[\left\|C x_{N-q+1}\right\|^{2}\right]\right. \\
=\sum_{l=1} \sigma_{N-q+1}^{S, l}+\sum_{l=1}^{2^{q-1}-1} \sigma_{N-q+1}^{K, l}+\sum_{l=1}^{2^{q-1}-2} \sigma_{N-q+1}^{C, l}+E\left[\left\|C x_{N-q+1}\right\|^{2}\right]
\end{gathered}
$$

where $V_{N-q+1, j}^{S, l}, V_{N-q+1, j}^{K, l}$ and $V_{N-q+1, j}^{C, l}$ are matrices of appropriate dimensions depending on the coefficients of the optimal control problem. We shall compute the optimal control $u_{N-q}$ and we shall prove that $\mathcal{O}\left(x_{0}, . . x_{N-q}\right)$ is given by a formula of the form (33) where $q$ is replaced by $q-1$.

We know that

$$
I_{N-q, x_{0}, . ., x_{N-q}}(u)=I_{N-q+1, x_{0}, . ., x_{N-q+1}}(u)+E\left[\left(\left\|C x_{N-q}\right\|^{2}+<K u_{N-q}, u_{N-q}>\right)\right] .
$$

Then

$$
\begin{gathered}
\min _{u_{N-q} \in \mathcal{U}_{N-q, N-q}^{a}}\left\{\min _{u_{N-q}, \ldots, u_{N-1} \in \mathcal{U}_{N-q, N-1}^{a}} I_{N-q, x_{0}, . ., x_{N-q+1}}(u)=\right. \\
\left.: \min _{u_{N-q+1}, \ldots, u_{N-1} \in \mathcal{U}_{N-q+1, N-1}^{a}} I_{x, N-q+1}(u)+E\left[\left(\left\|C x_{N-q}\right\|^{2}+\left\langle K u_{N-q}, u_{N-q}\right\rangle\right)\right]\right\} \\
:=\min _{u_{N-q} \in \mathcal{U}_{N-q, N-q}^{a}} f\left(x_{0}, . ., x_{N-q}, x_{N-q+1}, u_{N-q}\right) .
\end{gathered}
$$


Substituting

$$
x_{N-q+1}=\sum_{j=0}^{N-q} A_{j} x_{N-q-j}+\xi_{N-q} B x_{N-q}+D u_{N-q}+\xi_{N-q} F u_{N-q}
$$

in $\sigma_{N-q+1}^{S, l}($ see (33)) $)$ we obtain $\sigma_{N-q+1}^{S, l}$ as a function of the known $x_{j}, j=$ $0, N-q$ and the unknown $u_{N-q}$. We have

$$
\begin{gathered}
\sigma_{N-q+1}^{S, l}= \\
E\left\|\sqrt{S}\left(\sum_{j=1}^{N-q+1} V_{N-q+1, j}^{S, l} x_{N-q+1-j}+V_{N-q+1,0}^{S, l} \sum_{j=0}^{N-q} A_{j} x_{N-q-j}+V_{N-q+1,0}^{S, l} D u_{N-q}\right)\right\|^{2} \\
+E\left\|\sqrt{S} V_{N-q+1,0}^{S, l}\left(B x_{N-q}+F u_{N-q}\right)\right\|^{2} \\
=E\left\|\sqrt{S} \sum_{j=0}^{N-q}\left(V_{N-q+1, j+1}^{S, l}+V_{N-q+1,0}^{S, l} A_{j}\right) x_{N-q-j}\right\|^{2}+E\left\|\sqrt{S} V_{N-q+1,0}^{S, l} B x_{N-q}\right\|^{2} \\
+2 E<S \sum_{j=0}^{N-q}\left(V_{N-q+1, j+1}^{S, l}+V_{N-q+1,0}^{S, l} A_{j}\right) x_{N-q-j}, V_{N-q+1,0}^{S, l} D u_{N-q}> \\
\left.+2 E\left\langle\left(V_{N-q+1,0}^{S, l}\right)^{*} S V_{N-q+1,0}^{S, l} B x_{N-q}, F u_{N-q}\right)\right\rangle \\
+E\left\|\sqrt{S} V_{N-q+1,0}^{S, l} D u_{N-q}\right\|^{2}+E\left\|\sqrt{S} V_{N-q+1,0}^{S, l} F u_{N-q}\right\|^{2}
\end{gathered}
$$

A similar computation leads to a formula for $\sigma_{N-q+1}^{K}$ and $\sigma_{N-q+1}^{C}$ obtained from the one above by replacing $S$ by $K$. Also

$$
\begin{gathered}
E\left[\left\|C x_{N-q+1}\right\|^{2}\right]=E\left\|C \sum_{j=0}^{N-q} A_{j} x_{N-q-j}\right\|^{2}+E\left\|C B x_{N-q}\right\|^{2}+ \\
2 E<D^{*} C^{*} C \sum_{j=0}^{N-1} A_{j} x_{N-q-j}, u_{N-q}>+2 E\left\langle F^{*} C^{*} C B x_{N-q}, u_{N-q}\right\rangle \\
+E\left\|C F u_{N-q}\right\|^{2}+E\left\|C D u_{N-q}\right\|^{2} .
\end{gathered}
$$

Therefore, substituting (34) in $f\left(x_{0}, . ., x_{N-q}, x_{N-q+1}, u_{N-q}\right)$ we see that $f\left(x_{0}, . ., x_{N-q}, x_{N-q+1}, u_{N-q}\right)$ becomes a function $g\left(x_{0}, . ., x_{N-q}, u_{N-q}\right)$ of the form

$$
w_{N-q}\left(x_{0}, . ., x_{N-q}\right)+2 E\left\langle v_{N-q}\left(x_{0}, . ., x_{N-q}\right), u_{N-q}\right\rangle+E\left\langle J_{N-q} u_{N-q}, u_{N-q}\right\rangle,
$$


where

$$
\begin{gathered}
J_{N-q}=K+\sum_{l=1}^{2^{q-1}}\left[\left(V_{N-q+1,0}^{S, l} D\right)^{*} S V_{N-q+1,0}^{S, l} D+\left(V_{N-q+1,0}^{S, l} F\right)^{*} S V_{N-q+1,0}^{S, l} F\right] \\
+\sum_{l=1}^{2^{q-1}-1}\left[\left(V_{N-q+1,0}^{K, l} D\right)^{*} K V_{N-q+1,0}^{K, l} D+\left(V_{N-q+1,0}^{K, l} F\right)^{*} K V_{N-q+1,0}^{K, l} F\right] \\
+\sum_{l=1}^{2^{q-1}-2}\left[\left(V_{N-q+1,0}^{C, l} D\right)^{*} C^{*} C V_{N-q+1,0}^{C, l} D+\left(V_{N-q+1,0}^{C, l} F\right)^{*} C^{*} C V_{N-q+1,0}^{C, l} F\right] \\
+F^{*} C^{*} C F+D^{*} C^{*} C D>0
\end{gathered}
$$

and

$$
\begin{gathered}
v_{N-q}\left(x_{0}, \ldots, x_{N-q}\right)= \\
\sum_{l=1}^{2^{q-1}}\left\{\left(V_{N-q+1,0}^{S, l} D\right)^{*} S \sum_{j=0}^{N-q}\left(V_{N-q+1, j+1}^{S, l}+V_{N-q+1,0}^{S, l} A_{j}\right) x_{N-q-j}+\right. \\
\left.\left(V_{N-q+1,0}^{S, l} F\right)^{*} S V_{N-q+1,0}^{S, l} B x_{N-q}\right\}+ \\
\sum_{l=1}^{2^{q-1}-1}\left\{\left(V_{N-q+1,0}^{K, l} D\right)^{*} K \sum_{j=0}^{N-q}\left(V_{N-q+1, j+1}^{K, l}+V_{N-q+1,0}^{K, l} A_{j}\right) x_{N-q-j}+\right. \\
\left.\left(V_{N-q+1,0}^{K, l} F\right)^{*} K V_{N-q+1,0}^{K, l} B x_{N-q}\right\}+ \\
\sum_{l=1}^{2^{q-1}-2}\left\{\left(V_{N-q+1,0}^{C, l} D\right)^{*} C^{*} C \sum_{j=0}^{N-q}\left(V_{N-q+1, j+1}^{C, l}+V_{N-q+1,0}^{C, l} A_{j}\right) x_{N-q-j}+\right. \\
\left.\left(V_{N-q+1,0}^{C, l} F\right)^{*} C^{*} C V_{N-q+1,0}^{C, l} B x_{N-q}\right\}+ \\
D^{*} C^{*} C \sum_{j=0}^{N-q} A_{j} x_{N-q-j}+F^{*} C^{*} C B x_{N-q} .
\end{gathered}
$$

Reasoning as in the case $q=1$, we get the optimal control

$$
\begin{aligned}
u_{N-q}^{*}\left(x_{0}, . ., x_{N-q}\right) & =-J_{N-q}^{-1} v_{N-q} \\
& =\sum_{j=0}^{N-q} W_{j, N-q} x_{N-q-j} .
\end{aligned}
$$

Taking into account (36), we see that for all $j \in\{0,1, \ldots, N-q\}, W_{j, N-q}$ is obtained by multiplying the coefficient of $x_{N-q-j}$, from $v_{N-q}$, with $-J_{N-q}^{-1}$. 
Replacing (37) in $\sigma_{N-q+1}^{S, l}$, we observe that, for all $l \in\left\{1, . ., 2^{q-1}\right\}$,

$$
\begin{gathered}
\sigma_{N-q+1}^{S, l}= \\
E\left\|S \sum_{j=0}^{N-q}\left(V_{N-q+1, j+1}^{S, l}+V_{N-q+1,0}^{S, l} A_{j}\right) x_{N-q-j}+V_{N-q+1,0}^{S, l} D u_{N-q}\right\|^{2}+ \\
+E\left\|\sqrt{S} V_{N-q+1,0}^{S, l}\left(B x_{N-q}+F u_{N-q}\right)\right\|^{2} \\
=E\left\|\sqrt{S} \sum_{j=0}^{N-q} V_{N-q, j}^{S, l} x_{N-q-j}\right\|^{2}+E\left\|\sqrt{S} \sum_{j=0}^{N-q} V_{N-q, j}^{S, l+2^{q-1}} x_{N-q-j}\right\|^{2}
\end{gathered}
$$

and

$$
\sum_{l=1}^{2^{q-1}} \sigma_{N-q+1}^{S, l}=\sum_{l=1}^{2^{q}} E\left\|\sqrt{S} \sum_{j=0}^{N-q} V_{N-q, j}^{S, l} x_{N-q-j}\right\|^{2},
$$

where

$$
\begin{aligned}
V_{N-q, j}^{S, l} & =V_{N-q+1, j+1}^{S, l}+V_{N-q+1,0}^{S, l}\left(A_{j}+D W_{j, N-q}\right), \\
V_{N-q, 0}^{S, l+2^{q-1}} & =V_{N-q+1,0}^{S, l}\left(B+F W_{0, N-q}\right), \\
V_{N-q, j}^{S, l+2^{q-1}} & =V_{N-q+1,0}^{S, l} F W_{j, N-q}, j \neq 0 .
\end{aligned}
$$

Arguing as above and using (29), we obtain

$$
\begin{aligned}
\sum_{l=1}^{2^{q-1}-1} E[\| \sqrt{K} & \sum_{j=0}^{N-q+1} V_{N-q+1, j}^{K, l} x_{N-q+1-j} \|^{2}+\left\langle K u_{N-q}, u_{N-q}\right\rangle= \\
& =\sum_{l=1}^{2^{q}-1} E\left\|\sqrt{K} \sum_{j=0}^{N-q} V_{N-q, j}^{K, l} x_{N-q-j}\right\|
\end{aligned}
$$

where for all $l \in\left\{1, \ldots, 2^{q-1}-1\right\}, j \leq N-q$

$$
\begin{aligned}
V_{N-q, j}^{K, l} & =V_{N-q+1, j+1}^{K, l}+V_{N-q+1,0}^{K, l}\left(A_{j}+D W_{j, N-q}\right), \\
V_{N-q, 0}^{K, l+2^{q-1}-1} & =V_{N-q+1,0}^{K, l}\left(B+F W_{0, N-q}\right), \\
V_{N-q, j}^{K, l+2^{q-1}-1} & =V_{N-q+1,0}^{K, l} F W_{j, N-q}, V_{N-q, j}^{K, 2^{q}-1}=W_{j, N-q}, j \neq 0 .
\end{aligned}
$$

Similarly,

$$
\begin{gathered}
\sum_{l=1}^{2^{q-1}-2} E\left[\left\|C \sum_{j=0}^{N-q+1} V_{N-q+1, j}^{K, l} x_{N-q+1-j}\right\|^{2}+E\left[\left\|C x_{N-q+1}\right\|^{2}\right]^{2}=\right. \\
\quad \sum_{l=1}^{2^{q}-2} E\left\|C \sum_{j=0}^{N-q} V_{N-q, j}^{C, l} x_{N-q-j}\right\|
\end{gathered}
$$


where $V_{N-q, j}^{C, l}, 1 \leq l \leq 2^{q-1}-2, j \leq N-q$ are given by

$$
\begin{aligned}
V_{N-q, j}^{C, l} & =V_{N-q+1, j+1}^{C, l}+V_{N-q+1,0}^{C, l}\left(A_{j}+D W_{j, N-q}\right), \\
V_{N-q, 0}^{C, l+2^{q-1}-2} & =V_{N-q+1,0}^{C, l}\left(B+F W_{0, N-q}\right), \\
V_{N-q, j}^{C, l+2^{q-1}-2} & =V_{N-q+1,0}^{C, l} F W_{j, N-q}, V_{N-q, j}^{C, 2^{q}-3}=A_{j}+D W_{j, N-q}, j \neq 0 \\
V_{N-q, 0}^{C, 2^{q}-2} & =B+F W_{0, N-q}, V_{N-q, j}^{C, 2^{q}-2}=F W_{j, N-q}, j \neq 0 .
\end{aligned}
$$

Now it is clear that a formula for $\mathcal{O}\left(x_{0}, . ., x_{N-q}\right)$ can be obtained by replacing $q$ with $q+1$ in (33) and using the coefficients (31), (32), (38), (39) and (40).

The optimal cost $I_{x_{0}, N}(u)$ is given by $\mathcal{O}\left(x_{0}\right)$, i.e. by formula (33) written for $q=N+1$.

\section{References}

[1] O.P. Agrawal, A general formulation and solution scheme for fractional optimal control problems, Nonlinear Dynamics, 38, 1(2004), 323-337.

[2] O.P. Agrawal, A quadratic numerical scheme for fractional optimal control problems, Journal of Dynamic Systems, Measurement, and Control 130,1 (2008),1-6.

[3] A. Amirdjanova, S. Chivoret, New method for optimal nonlinear filtering of noisy observations by multiple stochastic fractional integral expansions, Computers \& Mathematics with Applications 52, 1 (2006), 161-178.

[4] B. D. A. Anderson, J. B. Moore, Optimal control: linear quadratic methods, Prentice Hall, Englewood Cliffs, 1990.

[5] M. Athans, P. L.Falb, Optimal Control: An Introduction to the Theory and Its Applications, Dover Publications, New York, 2006.

[6] B. Bandyopadhyay, S. Kamal, Stabilization and Control of Fractional Order Systems: A Sliding Mode Approach, Springer International Publishing, Switzerland, 2015.

[7] O. L.V. Costa, M. D. Fragoso, M. G. Todorov, Continuous-Time Markov Jump Linear Systems, Springer Berlin Heidelberg, 2013.

[8] V. Dragan, T. Morozan, A. Stoica, Mathematical Methods in Robust Control of Discrete Time Linear Stochastic Systems, Springer, New York, 2010.

[9] A. Dzielinski,P. M. Czyronis, Dynamic Programming for Fractional Discrete-time Systems, $19^{\text {th }}$ World Congress of IFAC,19(2014), 1.

[10] R. Kamocki, M. Majewski, Fractional linear control systems with Caputo derivative and their optimization, Optimal Control Applications and Methods 36.6 (2015): 953-967. 
[11] U.N.Katugampola, A New Approach To Generalized Fractional Derivatives, Bull. Math. Anal. App. 6(2014), 4, 1-15.

[12] G. Idiri, S. Djennounet, M. Bettayeb, Solving fractional optimal control problems using control vector parameterization, Control, Decision and Information Technologies (CoDIT), 2013 International Conference on. IEEE, 2013.

[13] F. Mainardi, Fractional Calculus and Waves in Linear Viscoelasticity: An Introduction to Mathematical Models, World Scientific, Singapore, 2010.

[14] K. S. Miller, B. Ross, An introduction to the fractional calculus and fractional differential equations, Wiley-Interscience Publication, New York, 1993.

[15] C.A. Monje et al. Fractional-order Systems and Controls: Fundamentals and Applications, Springer Science \& Business Media, London, 2010.

[16] H. Sadeghian, et al. On the general Kalman filter for discrete time stochastic fractional systems, Mechatronics 23.7 (2013): 764-771.

[17] J.I. Suárez, B. M. Vinagre, Y. Q. Chen, A fractional adaptation scheme for lateral control of an $A G V$, Journal of Vibration and Control 14.9-10 (2008): 1499-1511.

[18] C. Tricaud, Y. Q. Chen. An approximate method for numerically solving fractional order optimal control problems of general form, Computers \& Mathematics with Applications 59, 5(2010), 1644-1655.

[19] V. M. Ungureanu, Stability, stabilizability and detectability for Markov jump discrete-time linear systems with multiplicative noise in Hilbert spaces, Optimization 63.11 (2014): 1689-1712

[20] V. M. Ungureanu, V. Dragan, T. Morozan, Global solutions of a class of discrete-time backward nonlinear equations on ordered Banach spaces with applications to Riccati equations of stochastic control, Optimal Control Applications and Methods, 34(2013), 2, 164-190.

[21] M. Wojciech, J. Kacprzyk,J. Baranowski. Advances in the Theory and Applications of Non-integer Order Systems, 5th Conference on Non-integer Order Calculus and Its Applications, Cracow, Poland. Lecture Notes in Electrical Engineering, 257, 2013. 